Published online 2017 April 13.

Abstract

\title{
The Current Diagnostic and Treatment Options for Managing
}

\section{Hemobilia}

\section{Mohammad Gharib Salehi ${ }^{1, *}$}

${ }^{1}$ Assistant Professor of Medical Science, Imam Reza Hospital, Kermanshah University of Medical Science, Kermanshah, Iran

"Corresponding author: Mohammad Gharib Salehi, MD, Assistant Professor of Medical Science, Imam Reza Hospital, Kermanshah University of Medical Science, Kermanshah, Iran. E-mail: Kumssalehi@yahoo.com

Received 2016 December 21; Accepted 2017 February 08.

\begin{abstract}
hemobilia is a rare source of upper gasterointestinal bleeding. Though the incidence is increasing along with the rise in minimally invasive biliary interventions. Prompt diagnosis and treatment rests of having appropriate clinical Suspicion which should be based on the patients presenting signs and symptoms, as well as history including recent instrumentation. Endoscopy should be reservedfor cases of upper gasterointestinal bleeding with low suspicion for hemobilia. Interventional radiology may be the first line diagnostic and therapeutic option for patients with a high suspicion of hemobilia. While embolization is the mainstay of therapy, other options include thrombin injection, stent placement, and/or placement of a percutaneous biliary drain. Surgery should be reserved for failed treatment by interventional radiologist.
\end{abstract}

This is an abstract presented in the 33rd Iranian congress of radiology (ICR) and the 15th congress of Iranian radiographic science association (IRSA). 